\title{
Thickness control of the silica shell: a way to tune the plasmonic properties of isolated and assembled gold nanorods
}

\author{
Angela Candreva $\cdot$ Wiktor Lewandowski • \\ Massimo La Deda
}

Received: 10 September 2021 / Accepted: 6 January 2022 / Published online: 19 January 2022

(C) The Author(s) 2022

\begin{abstract}
By combining photophysical measurements with transmission electron microscopy, we proved that the thickness of the silica shell around gold nanorods determines the position of the longitudinal plasmonic band when they are isolated in solution or assembled in solid. The silica thickness has been tuned by modulating the reaction time and the ratio between CTAB-coated gold nanorods and TEOS concentration, obtaining gold nanorods covered by a silica shell with a thickness varying from 3.5 to 24 $\mathrm{nm}$. Considering this shell as a spacer between the gold cores, it is possible to modulate the coupling of the localized surface plasmon resonance (LSPR) of neighboring nanorods. Moreover, the comparison between the extinction spectra in solution and in solid, recorded from nanorods covered by silica shell with different thickness, can be used to estimate the
\end{abstract}

Supplementary Information The online version contains supplementary material available at https://doi. org/10.1007/s11051-022-05402-w.

A. Candreva $\cdot$ M. La Deda $(\bowtie)$

Department of Chemistry and Chemical Technologies, University of Calabria, 87036 Rende, CS, Italy

e-mail: massimo.ladeda@unical.it

A. Candreva $\cdot$ M. La Deda

CNR-NANOTEC, Istituto di Nanotecnologia U.O.S, Cosenza, 87036 Rende, CS, Italy

W. Lewandowski

Faculty of Chemistry, University of Warsaw, 1 Pasteura St, 02-093 Warsaw, Poland inter-nanoparticles distance required for plasmon interaction. We found that LSPR coupling is effective when the distance between the gold cores is no more than $10 \mathrm{~nm}$. When the distance is greater, the nanorods do not interact with each other.

Keywords Gold nanorods $\cdot$ Silica thickness $\cdot$ LSPR band $\cdot$ Plasmon coupling

\section{Introduction}

Gold nanoparticles (AuNPs) with various shapes, size, and organization show unique scattering spectra depending on their geometries (Hao et al. 2004; Xia et al. 2003; Creighton and Eadon 1991; Candreva et al. 2020). Spectroscopy and TEM are precious techniques to identify, characterize, and study nanoparticle features. Anisotropic nanoparticles show more than one plasmonic peak located in the visible and near-infrared region of the electromagnetic spectrum, associated with the various orientations of the particle axes relative to the electric field of light (Hao et al. 2004; Eustis and El-Sayed 2006; Burda et al. 2005; Teranishi et al. 2000; Yamada and Niidome 2006). In particular, for gold nanorods (AuNRs), the transverse oscillation is peaked at around $513 \mathrm{~nm}$ (Wu et al. 2016; Murphy et al. 2011), while the longitudinal resonance gives rise to a band whose position, ranging from 700 to $850 \mathrm{~nm}$, depends on the aspect ratio and other factors, such as the covering agent 
(Pérez-Juste et al. 2005; Chen et al. 2013; Wang et al. 2013a; Wang et al. 2020; Wu and Tracy 2015; Mayer and Hafner 2011). Organized superstructures of gold nanorods show an optical appearance different from the isolated nanorods (Zhang et al. 2015; Vial et al. 2007; Lunn et al. 2015), and this behavior has been attributed to the plasmon coupling (Jain et al. 2006).

To spatially organize anisotropic gold nanoparticles, two strategies should in principle be followed: one based on the interparticle forces due to a proper functionalization of the AuNPs (direct assembly) (Ni et al. 2010; Kumar et al. 2013; Kawamura et al. 2007; Ahijado-Guzmán et al. 2016); the other based on the presence of an orienting medium (indirect assembly) (Coursault et al. 2012; Rožič et al. 2017). In any case, plasmonic coupling was found to be highly dependent on interparticle spacing and the effectiveness of the NP coating in modulating the plasmonic field. Coverage can modulate the plasmonic field in several ways: it can guide the assembly of nanoparticles in a precise structural order, (Jain et al. 2006) or avoid plasmon interaction (Comenge et al. 2016) by increasing the distance between nanoparticles. The effectiveness of plasmonic coupling increases as the distance between the nanorods decreases and the number of interacting NPs increases. (Kumar et al. 2013; Sahu and Raj n.d.).

In this perspective, by using a versatile coating agent such as silica, it is possible to adjust the maximum distance between the nanoparticles necessary for the plasmon interaction. The thickness of the silica coating that covers AuNPs can be adjusted with the consequence of modulating the distance between the assembled gold nanorods and controlling their plasmonic coupling properties. With this in mind, the silica coating strategy (Wu and Tracy 2015; Jiang et al. 2016; Gorelikov and Matsuura 2008; Liz-Marzán et al. 1996; Carrasco et al. 2016; Guerrero-Martínez et al. 2010; Abadeer et al. 2014; Pellas et al. 2020) consists of a $\mathrm{pH}$-controlled condensation of tetraethylorthosilicate (TEOS) precursor onto CTAB-capped gold nanorods. Hydrolysis and condensation of TEOS require alkaline conditions, generally reached by adding to the sample ammonia or strong bases such as $\mathrm{NaOH}$. CTAB surfactant deposited onto AuNRs is a structure-directing agent (Ming et al. 2009) that facilitates the formation of mesostructured silica shell around nanorods. The silica shell grows anisotropically on AuNRs; it is deposited first selectively at the two ends of the nanorods, due to the greater curvature, then grows over the entire surface of the nanoparticles (Adelt et al. 2021; Wang et al. 2013b).

The synthesis of silica-coated AuNRs requires fine-tuning the value of some parameters and the quantities of the reagents: here the individual contributions are discussed, estimating the quantity of each to obtain a homogeneous silica shell. Considering the reaction time and the ratio between the concentrations of the CTAB-coated AuNRs and TEOS, we adjusted the thickness of the silica around NRs. Using this cover agent as spacer between AuNRs, we found that the coupling of the plasmonic fields is effective when the distance between nanoparticles is not higher than $10 \mathrm{~nm}$. When the distance is greater, no nanorod interact each other.

\section{Materials and methods}

\section{Chemicals}

Hexadecyltrimethylammonium bromide (CTAB, $\geq 96 \%$ ), 5-bromosalicylic acid (technical grade, 90\%), hydrogen tetrachloroaurate trihydrate $\left(\mathrm{HAuCl}_{4} \bullet \mathrm{H}_{2} \mathrm{O}\right.$, $\geq 99.9 \%)$, silver nitrate $\left(\mathrm{AgNO}_{3}, \geq 99.0 \%\right)$, L-ascorbic acid ( $\geq 99 \%$ ), sodium iodide $99.99 \%)$, trisodium citrate (99\%), polyvinilpolypyrrolidone (PVP K15, Mn $10000)$, and sodium borohydride $\left(\mathrm{NaBH}_{4}, 99 \%\right)$ were purchased from Aldrich and used as received. MilliQ water (resistivity $18.2 \mathrm{M} \Omega \bullet \mathrm{cm}$ at $25^{\circ} \mathrm{C}$ ) was used in all experiments. All glasswares were washed with aqua regia, rinsed with water, sonicated threefold for 3 min with Milli-Q water, and dried before use. TEOS (Alfa Aesar, 99.9\%), NaOH (Sigma Aldrich, 98\%), and $\mathrm{MeOH}$ (Sigma Aldrich) were used for the $\mathrm{SiO}_{2}$ over coating.

Instruments and methods

A Perkin Elmer Lambda 900 spectrophotometer was employed to obtain the extinction spectra (Cretu et al. 2018; Pf et al. 2020; Ionescu et al. 2019). The size and morphology of the gold nanoparticles were measured using a transmission electron microscope (Jeol JEM-1400 Plus $120 \mathrm{kV}$ ). The samples for transmission electron microscopy (TEM) were prepared by depositing a drop of a diluted solution on 300 mesh copper grids. After evaporation of the solvent in air at 
room temperature, the particles were observed at an operating voltage of $80 \mathrm{kV}$.

Synthesis

An aqueous solution of CTAB (until a final concentration of $1.0 \mathrm{E}-3 \mathrm{M}$ ) has been added to $2 \mathrm{~mL}$ of an aqueous dispersion of AuNR@CTAB (0.3 E-3 M, A.R. 4) (Cretu et al. 2018; Pf et al. 2020). After few seconds, $20 \mu \mathrm{L}$ of a $0.1 \mathrm{M} \mathrm{NaOH}$ aqueous solution were added under vigorous stirring, reaching a $\mathrm{pH}$ value of 8.5 , followed by three additions, in intervals of $1 \mathrm{~h}$ from each other, each of $12 \mu \mathrm{L}$, of TEOS $20 \%$ $\mathrm{v} / \mathrm{v}$ in methanol under gentle stirring, at room temperature. After $14 \mathrm{~h}$, the mixture was centrifuged in MilliQ water twice at $6000 \mathrm{rpm}$ for $10 \mathrm{~min}$ and the sample was dispersed in $2 \mathrm{~mL}$ of MilliQ water $(\mathrm{Wu}$ and Tracy 2015; Jiang et al. 2016). Undesired corefree silica particles can be eliminated by the appropriate centrifugation step, but it is not unusual that a small fraction remains in the final sample.

This sample made up of AuNRs coated by silica, AuNR@SiO ${ }_{2}$, (hereinafter referred to as the Starting Sample) and the protocol followed to obtain it, are the point of reference with which to compare the obtained results.

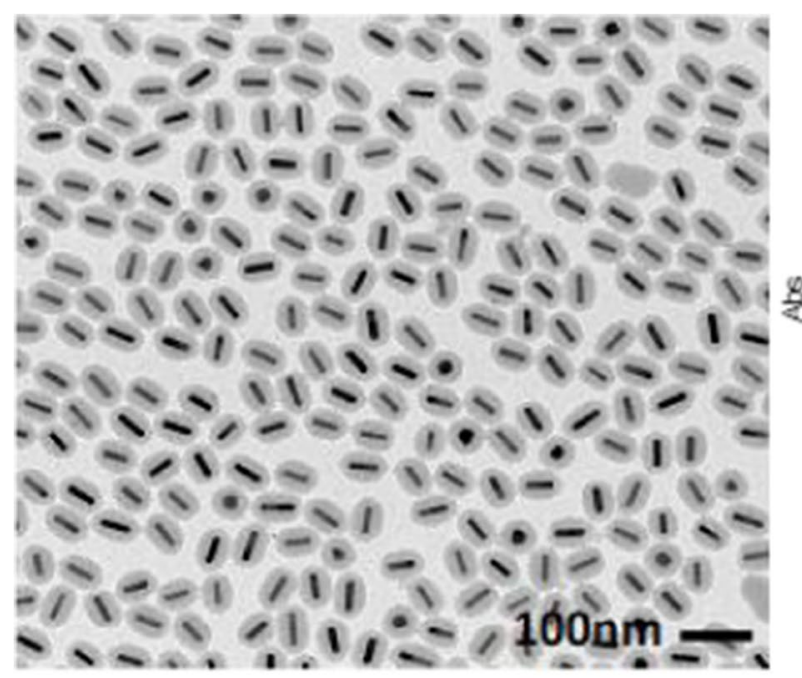

a

\section{Results and discussion}

Thickness control of silica shell and photophysical measurements of isolated gold nanorods

TEM image of the nanorods of the Starting Sample (Fig. 1a) shows the silica shell around nanorods with a thickness of $24 \mathrm{~nm} \times 12 \mathrm{~nm}$, long axis $\times$ short axis, respectively. Silica shell modifies the spectral position of the AuNRs longitudinal LSPR peak (Fig. 1b): the peak at $836 \mathrm{~nm}$ of AuNR@ $\mathrm{SiO}_{2}$ solution is redshifted compared to that of the AuNR@CTAB solution, positioned at $783 \mathrm{~nm}$. This is due to the fact that the metal-dielectric interface in the case of AuNR@ $\mathrm{SiO}_{2}$ is made up of silica which has a higher refractive index $\left(\mathrm{nd}^{20}=1.45\right)$ than the AuNR@CTAB interface, which is actually made up of the water $\left(\mathrm{nd}^{20}\right.$ $=1.33$ ) embedding CTAB. As shown below, as the thickness of the silica shell increases, the longitudinal band shifts towards red accordingly.

Experimental parameters controlled during the silica coverage of gold nanorods include $\mathrm{pH}$, concentration of the added CTAB solution, TEOS amount, concentration of the AuNR@CTAB solution, and the reaction time. Firstly, we have optimized the $\mathrm{pH}$ value and CTAB concentration, to obtain a detectable and homogeneous silica shell, and then we have studied

Fig. 1 Starting sample. a TEM image of AuNR@ $\mathrm{SiO}_{2}$. b Extinction spectra of AuNR@CTAB (red line; $\left.\lambda_{\mathrm{LSPR}}=783 \mathrm{~nm}\right)$ and AuNR@ $\mathrm{SiO}_{2}$ (black line; $\lambda_{\mathrm{LSPR}}=836 \mathrm{~nm}$ ) in water solution 
the correlation between the $[\mathrm{TEOS}] /\left[\mathrm{AuNR} @ \mathrm{SiO}_{2}\right]$ ratio and the silica coverage.

\section{Hydrolysis and condensation: $\mathrm{pH}$ value}

The quantity of $\mathrm{NaOH}$ necessary to obtain the Starting Sample corresponds to a $\mathrm{pH}$ value of 8.5. By changing the amount of $\mathrm{NaOH}$ to measure a $\mathrm{pH}$ value of around 10, the gold nanorods precipitate to the bottom of the vials in seconds. By lowering the amount of $\mathrm{NaOH}$ to reach a final $\mathrm{pH}$ value of 8 , no precipitation was recorded, but the TEM image (Fig. 1 in SI) showed the absence of an appreciable silica coating.

In the first case $(\mathrm{pH}=10)$, the alkalinity of the reaction environment causes a rapid TEOS polymerization creating a sort of cloud that incorporates a number of gold nanorods, triggering their precipitation. In the second case $(\mathrm{pH}=8)$, the alkalinity of the reaction environment is insufficient to prompt a good TEOS polymerization, so the silica coverage of nanorods is poor. In conclusion, $\mathrm{pH}=8.5$ can be considered an optimal value.

\section{Homogeneity of the silica shell: CTAB concentration}

As previously reported, the first step of the silica shell growth is the addition of an aqueous solution of CTAB to an aqueous dispersion of AuNR@CTAB. In the Starting Sample (see experimental part), we used a solution of CTAB $1.0 \mathrm{E}-3 \mathrm{M}$, but we ascertained that using a CTAB concentration of $5.0 \mathrm{E}-4 \mathrm{M}$, the silica coverage appears to be unchanged (Fig. 2 in SI). By halving the CTAB concentration again down to $2.5 \mathrm{E}-4 \mathrm{M}$, we obtained a disappointing result as shown in the TEM image of Fig. 3 in SI, while reducing again the CTAB concentration down to $2.5 \mathrm{E}-5$ $\mathrm{M}$, after few hours from the latest TEOS addition, a burgundy cloud appeared in the solution which in a few seconds precipitated on the bottom of the vials.

Because CTAB molecules adsorbed on AuNRs act as templates for the growth of the silica shell, it is necessary to maintain this coverage during TEOS condensation; nevertheless, the addition of $\mathrm{NaOH}$ can dislodge CTAB molecules from the gold surface (on which they are held by electrostatic interactions) due to the presence of the $\mathrm{Na}^{+}$hard ions.

Successively, we increased CTAB concentration up to 4.0E-3 M. TEM images (Fig. 4 in SI) show the expected AuNR@ $\mathrm{SiO}_{2}$ nanoparticles, but the silica coverage is not uniform on the gold surface, and the size of AuNR@ $\mathrm{SiO}_{2}$ appears inhomogeneous. These features can be attributed to the excessive CTAB concentration over the CMC (Scarabelli et al. 2015) that hampers the formation of the templating micelles, leading to a disordered silica growth.

\section{Thickness control: TEOS concentration}

The silica shell thickness can be modulated by varying the amount of TEOS. On this basis, we carry out various syntheses keeping constant the concentration of AuNR@CTAB (0.3E-3 M) and the other parameters (as reported in the paragraph Synthesis), and varying only the quantity of TEOS.

We tested four protocols with different amounts of TEOS, obtaining AuNR@ $\mathrm{SiO}_{2}$ samples with different thicknesses of silica determined by TEM (Table 1): Figs. 2, 3, 4, and 5 report TEM images, Fig. 6 the extinction spectra of the samples.

The silica thickness is reduced by decreasing the quantity of TEOS, although a considerable reduction of TEOS does not correspond to a notable thickness reduction. The extinction spectra show the typical red-shift due to the presence silica cover.

Table 1 Thickness control: TEOS concentration

\begin{tabular}{lllll}
\hline Sample & $\begin{array}{l}\text { Withdrawn volume from } \\
\text { TEOS 20\% v/v solution... }\end{array}$ & $\begin{array}{l}\text {..corresponding to an } \\
\text { added TEOS amount of }\end{array}$ & Obtained silica thickness & $\begin{array}{l}\text { Longitudinal } \\
\text { LSPR band maxi- } \\
\text { mum }\end{array}$ \\
\hline 1 & & $0.5 \mathrm{E}-4 \mathrm{~mol}$ & $24 \mathrm{~nm} \times 12 \mathrm{~nm}$ & $836 \mathrm{~nm}$ \\
Starting Sample & $36 \mu \mathrm{L}$ & $0.3 \mathrm{E}-4 \mathrm{~mol}$ & $24 \mathrm{~nm} x 12 \mathrm{~nm}$ & $836 \mathrm{~nm}$ \\
2 & $24 \mu \mathrm{L}$ & $0.2 \mathrm{E}-4 \mathrm{~mol}$ & $22 \mathrm{~nm} \times 12 \mathrm{~nm}$ & $836 \mathrm{~nm}$ \\
3 & $12 \mu \mathrm{L}$ & $0.1 \mathrm{E}-4 \mathrm{~mol}$ & $16 \mathrm{~nm} \times 12 \mathrm{~nm}$ & $836 \mathrm{~nm}$ \\
4 & $4 \mu \mathrm{L}$ & $0.3 \mathrm{E}-5 \mathrm{~mol}$ & Not detectable & $830 \mathrm{~nm}$ \\
\hline
\end{tabular}




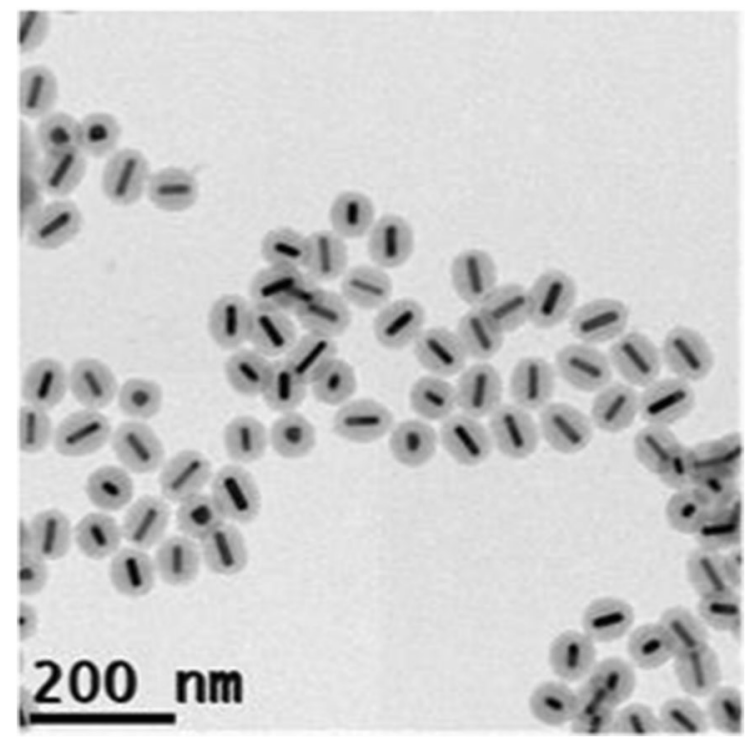

Fig. 2 TEM image from sample 1

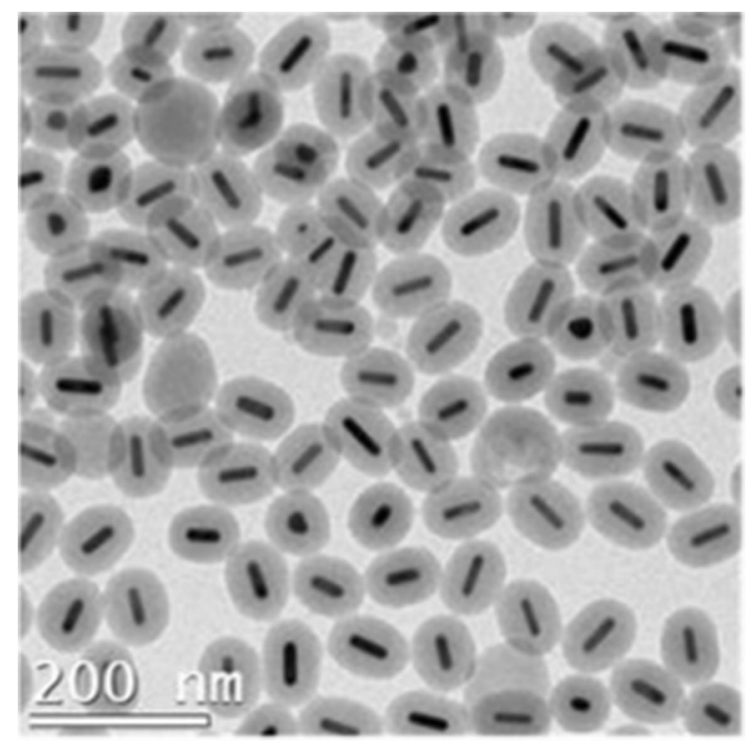

Fig. 3 TEM image from sample 2

It is interesting to note that the magnitude of the redshift is reduced by decreasing the amount of TEOS; this is to be attributed to a greater porosity of the shell, which incorporates a greater quantity of water.

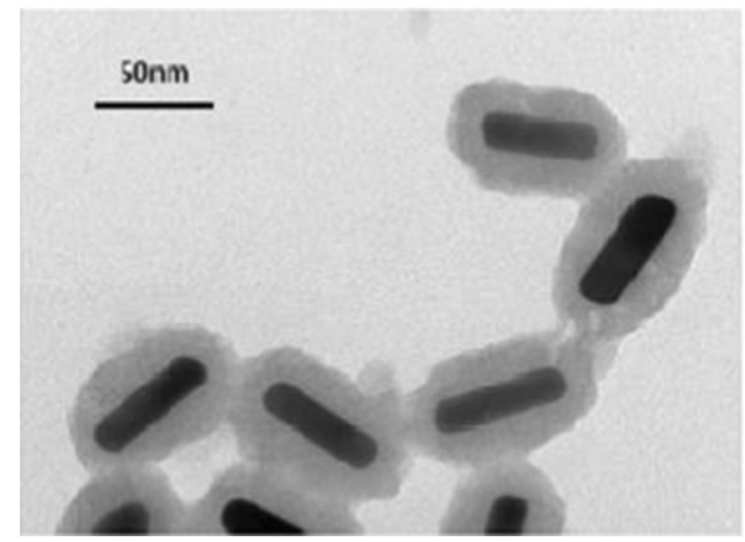

Fig. 4 TEM image from sample 3

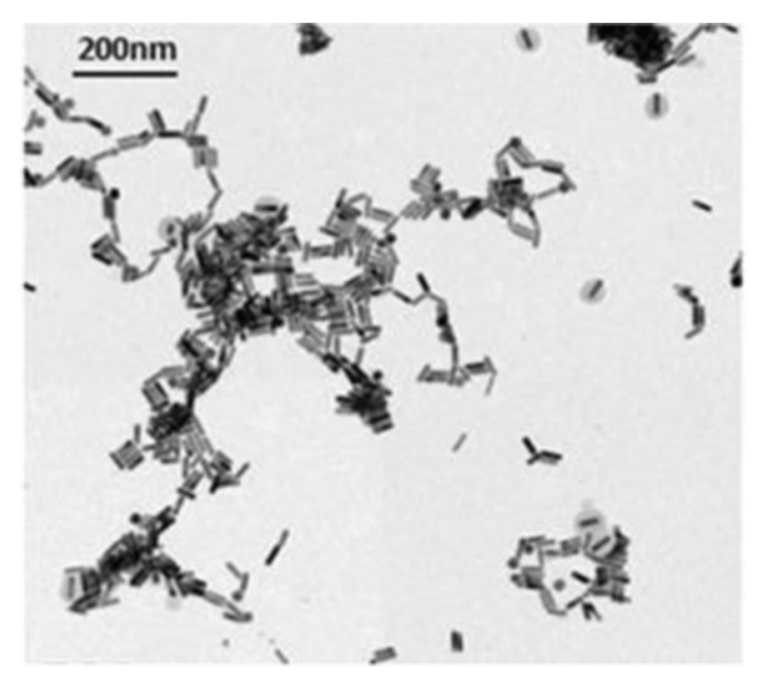

Fig. 5 TEM image from sample 4

\section{Thickness control:AuNR@CTAB concentration}

To test the effect of the AuNR@CTAB concentration on silica thickness, we increased this concentration up to 3.0E-3 M (Starting Sample' in Table 2). We estimated the AuNR concentration, according to Scarabelli et al., considering that all $\mathrm{Au}^{3+}$ has been reduced to $\mathrm{Au}^{0}$ (Scarabelli et al. 2015). By increasing the concentration of AuNR@CTAB, we correspondingly doubled the amount of $\mathrm{NaOH}$ to have a $\mathrm{pH}=8.5$. As expected, the thickness of the silica was significantly reduced compared to the products of the Starting Sample $(12 \mathrm{~nm} \times 7 \mathrm{~nm}$ vs $24 \mathrm{~nm} \times$ 


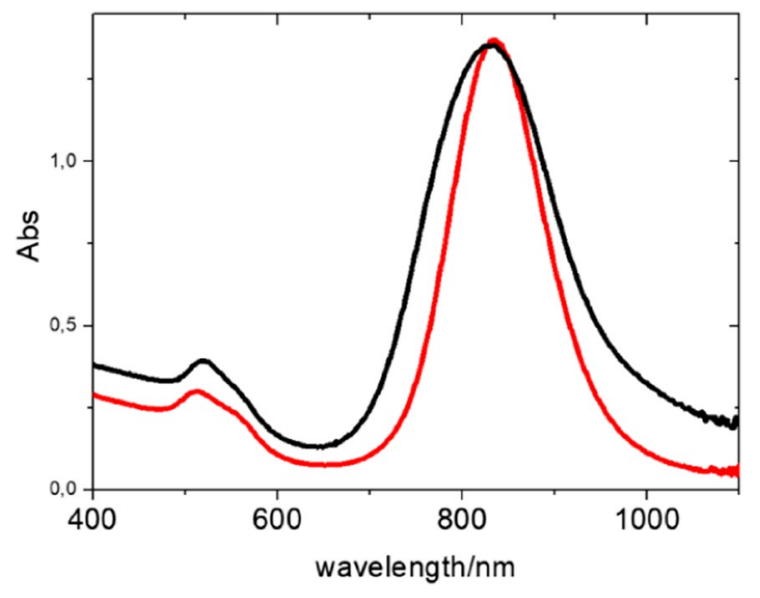

Fig. 6 Extinction spectra of the samples reported in Table 1: longitudinal LSPR peak of Starting Sample, 1, 2, 3 fall at the same wavelength (red line; $\lambda_{\text {LSPR }}=836 \mathrm{~nm}$ ) because the silica thickness does not change; extinction spectrum of sample 4 (black line; $\lambda_{\mathrm{LSPR}}=830 \mathrm{~nm}$ )

$12 \mathrm{~nm}$, respectively), because the amount of TEOS available for each AuNR is reduced.

Taking into account that TEOS amount used to obtain sample 3 (Table 1) allows to attain the thinner silica thickness, we fixed a TEOS amount of 0.1E-4 mol $(12 \mu \mathrm{L})$ (while Starting Sample sets a $0.3 \mathrm{E}-4$ mol value). Therefore, we prepared two samples, $3^{\prime}$ and 3", with the same TEOS amount (0.1E-4 mol, $12 \mu \mathrm{L})$ but different AuNR@CTAB concentration (Table 2), observing a different silica thickness: Fig. 7 $\mathrm{a}, \mathrm{b}, \mathrm{c}$ report TEM images of the samples, Fig. 7d the extinction spectra. In particular, spectra show the usual red-shift of the longitudinal NR band substituting CTB coverage with silica one, which is reduced according to the thinning of silica thickness.

Thickness control: reaction time

Silica shell growth is rapid during the first hour, but slows down considerably during the second hour of reaction. In the Starting Sample, the reaction time (i.e., the time interval between the last addition of TEOS and the purification step) was set at $14 \mathrm{~h}$. To study the effect of the reaction time variation on the silica thickness, we synthesized AuNR@ $\mathrm{SiO}_{2}$ samples starting from the three recipes already illustrated (Starting Sample, 3 and 3'), and reducing the reaction time to $1 \mathrm{~h}$.

The obtained results are reported in Table 3 and TEM images in Figures 8, 9, and 10.

Photophysical measurement of assembled gold nanorods covered with silica

To explore the occurrence of the coupling among AuNR@ $\mathrm{SiO}_{2}$, taking into account that the reciprocal distance between nanorods can be tuned by controlling the thickness of their silica shells, we compared the Starting Sample', 3',3". In particular, we compared extinction spectra from the water solution and from the film obtained by drop-casting of the solutions on quartz windows, thus, taking into account that in the solution there are no assemblies and therefore no coupling (Scarabelli et al. 2013), any difference should be attributable to the interaction of gold nanorods.

Observing the TEM image in Fig. 7a, we noticed the tendency of the nanoparticles to remain closed as much as possible, but no spectral variations were noted: the superimposition between the spectrum in solution and the spectrum of the film (Fig. 11) accounts for the absence of coupling. The small blue-shift of the longitudinal band of film sample compared to that in solution (hardly observable also in the previous sample), is attributed to the high sensitivity of this band to the refractive index variation on passing from water $\left(\mathrm{nd}^{20}=1.33\right)$ to air $\left(\mathrm{nd}^{20}\right.$ $=1.00)$.
Table 2 Thickness control: AuNR@CTAB concentration

\begin{tabular}{llll}
\hline Sample & [AuNR@CTAB]/M & Silica thickness & $\begin{array}{l}\text { Longitudinal } \\
\text { LSPR band maxi- } \\
\text { mum }\end{array}$ \\
\hline Starting Sample' & $3.0 \mathrm{E}-3(\mathrm{TEOS} 36 \mu \mathrm{L})$ & $12 \mathrm{~nm} \times 7 \mathrm{~nm}$ & $824 \mathrm{~nm}$ \\
$3^{\prime}$ & $3.0 \mathrm{E}-3(\operatorname{TEOS} 12 \mu \mathrm{L})$ & $10 \mathrm{~nm} \times 8 \mathrm{~nm}$ & $805 \mathrm{~nm}$ \\
$3^{\prime \prime}$ & $4.5 \mathrm{E}-3(\operatorname{TEOS~} 12 \mu \mathrm{L})$ & $5 \mathrm{~nm} \times 3.5 \mathrm{~nm}$ & $800 \mathrm{~nm}$ \\
\hline
\end{tabular}



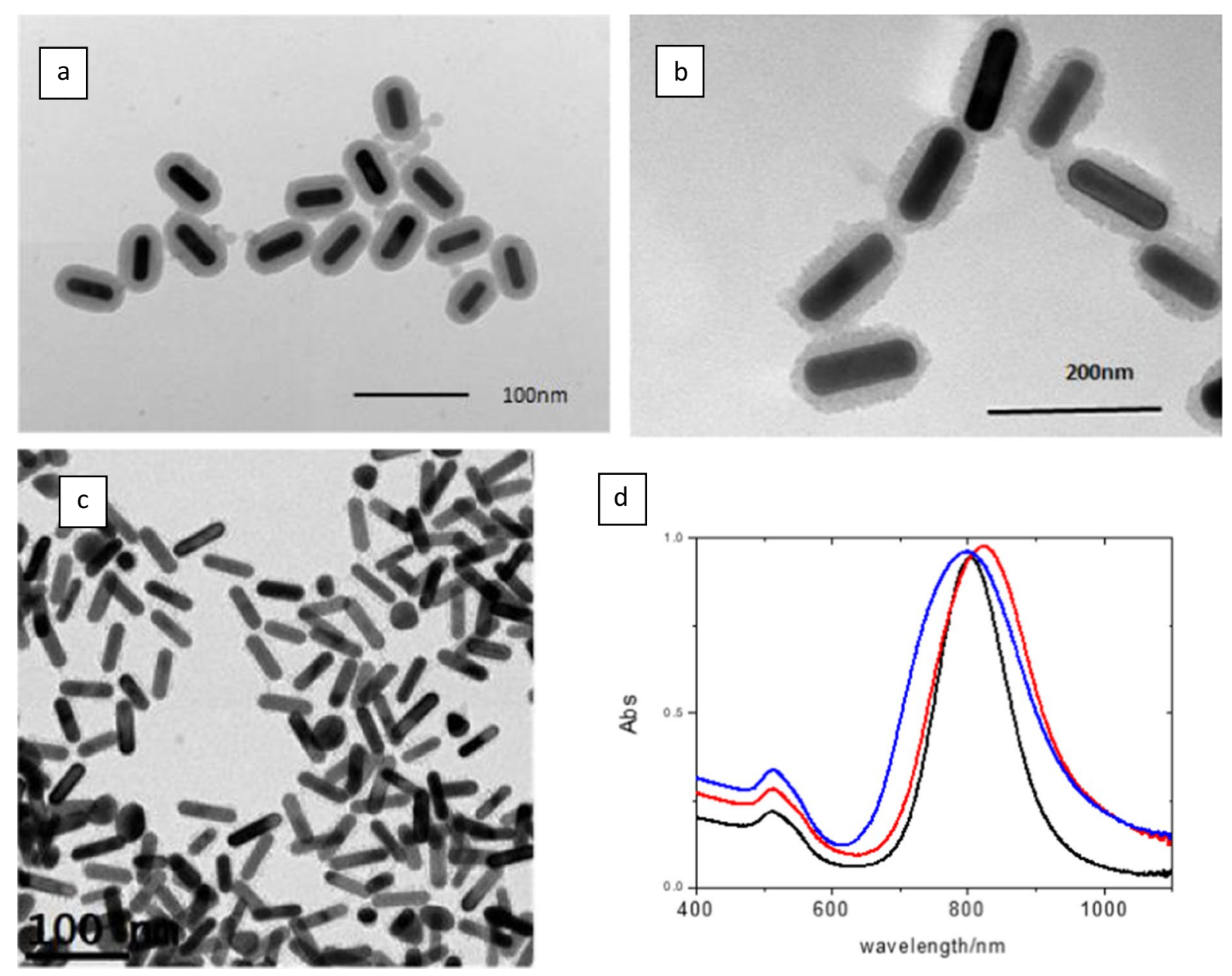

Fig. 7 a TEM image from Starting Sample'. b TEM image from sample 3'. c TEM image from sample 3". d Extinction spectra of Starting Sample' (red line; $\lambda_{\mathrm{LSPR}}=824 \mathrm{~nm}$ ), 3' (black line; $\left.\lambda_{\mathrm{LSPR}}=805 \mathrm{~nm}\right), 3^{\prime \prime}$ (blue line; $\lambda_{\mathrm{LSPR}}=800 \mathrm{~nm}$ )

Table 3 Thickness control: reaction time

\begin{tabular}{lll}
\hline Used recipe & $\begin{array}{l}\text { Silica thickness after } \\
\text { 14-h reaction time }\end{array}$ & $\begin{array}{l}\text { Silica thickness } \\
\text { after 1-h reaction } \\
\text { time }\end{array}$ \\
\hline Starting Sample & $24 \mathrm{~nm} \times 12 \mathrm{~nm}$ & $15 \mathrm{~nm} \times 13 \mathrm{~nm}$ \\
3 & $16 \mathrm{~nm} \times 12 \mathrm{~nm}$ & $13 \mathrm{~nm} \times 12 \mathrm{~nm}$ \\
$3^{\prime}$ & $10 \mathrm{~nm} \times 8 \mathrm{~nm}$ & $10 \mathrm{~nm} \times 6 \mathrm{~nm}$ \\
\hline
\end{tabular}

Sample 3' $10 \mathrm{~nm} \times 8 \mathrm{~nm}$

Observing the TEM images of Fig. 7b, we noticed that, due to the reduced thickness of the silica, the distance between the gold cores appears reduced. In this case, the spectrum of the solution and the spectrum

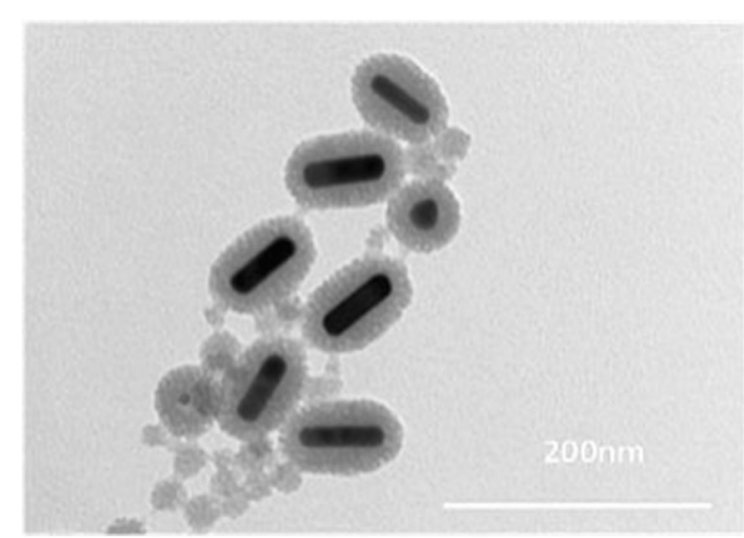

Fig. 8 TEM image of AuNR@ $\mathrm{SiO}_{2}$ prepared from the Starting Sample by reducing the reaction time. Unreacted silica present even after centrifugation is visible 


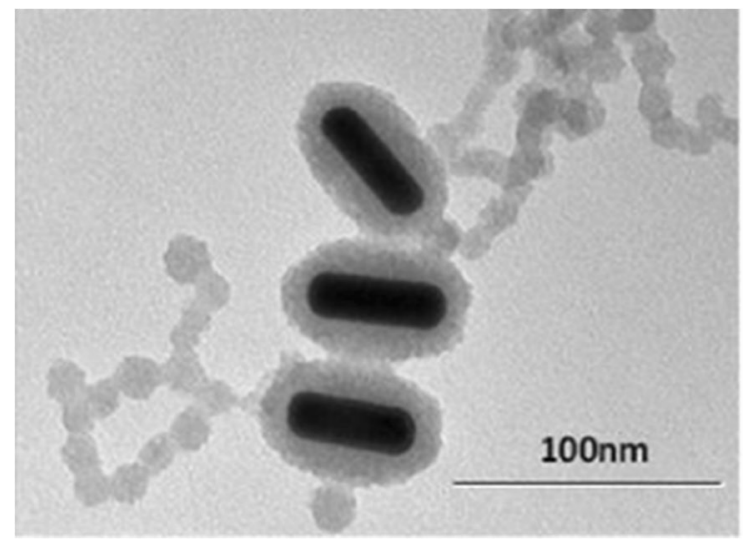

Fig. 9 TEM image of AuNR@ $\mathrm{SiO}_{2}$ prepared according to the recipe used to prepare sample 3

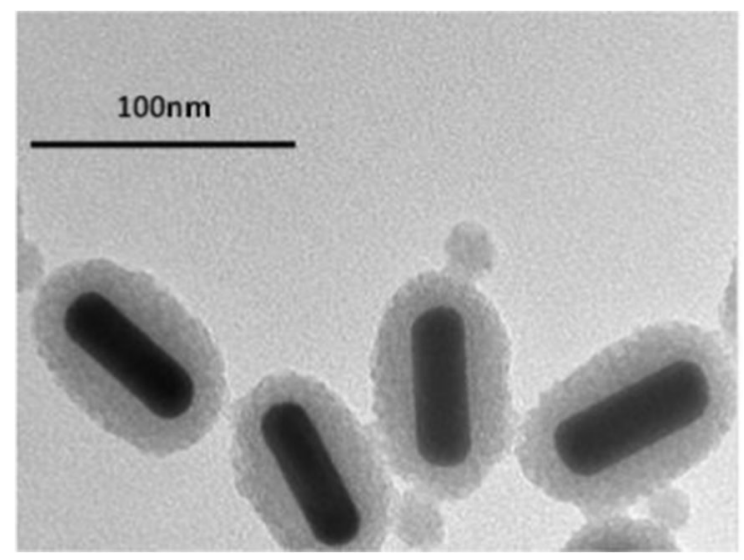

Fig. 10 TEM image of AuNR@ $\mathrm{SiO}_{2}$ prepared according to the recipe used to prepare sample $3^{\prime}$ of the film (Fig. 11) are different; in particular, the longitudinal plasmonic bands of the film spectrum are red-shifted and broadened with respect to those of the spectrum in solution. This behavior is attributed to the coupling of the plasmonic fields, allowed by the reduced distance between the gold cores.

\section{Sample 3" $5 \mathrm{~nm} \times 3.5 \mathrm{~nm}$}

Looking at the TEM images in Fig. 7c, the thickness of the silica shell is so thin that it is barely visible. The longitudinal plasmon band of the film spectrum (Fig. 11), with respect to the spectrum in solution, is red-shifted, but the band broadening in the film spectrum is impressive. This behavior is attributed to the coupling of the plasmonic fields, allowed by the reduced distance between the gold cores of the AuNRs due to a very thin silica shell of $5 \mathrm{~nm} \times 3.5$ nm.

\section{Conclusion}

Various parameters control the growth and thickness of silica shell of gold nanorods, but the obtained results show that the impact of the parameters is different, and small variations of someone can produce very important effects. For example, $\mathrm{pH}$ and CTAB concentration are difficult to modulate, while TEOS concentration is not very sensitive, because, as reported in Table 1, important variations of this parameter do not cause relevant thickness variations; on the contrary, the amount of AuNR@CTAB seems
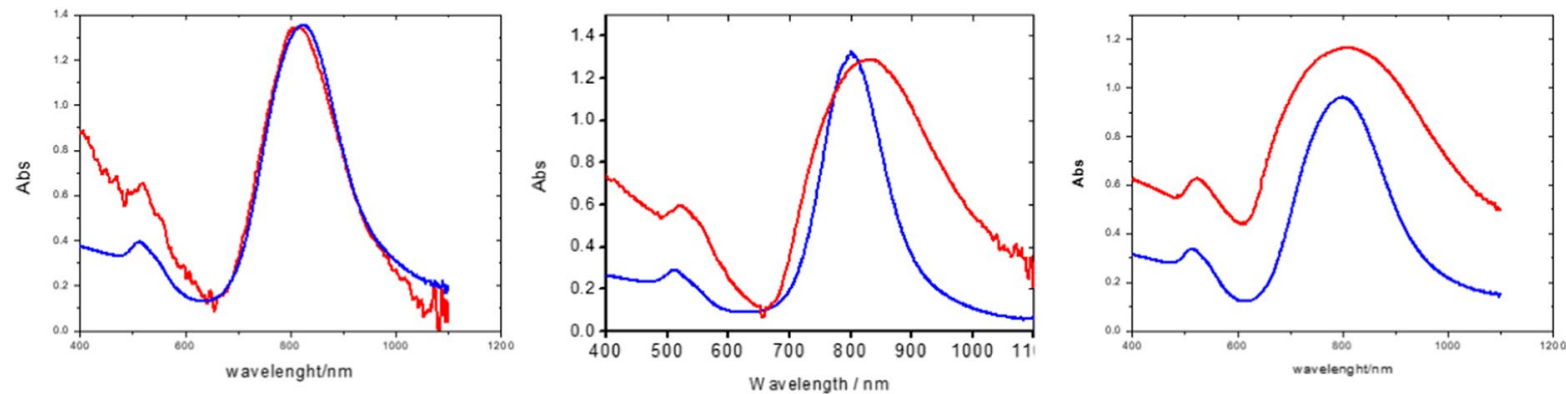

Fig. 11 From left to right: extinction spectrum of Starting Sample' from water solution (blue line), and from film sample (red line); extinction spectrum of $3^{\prime}$ from water solution (blue line, and from film sample (red line); extinction spectrum of $3^{\prime \prime}$ from water solution (blue line) and from film sample (red line) 


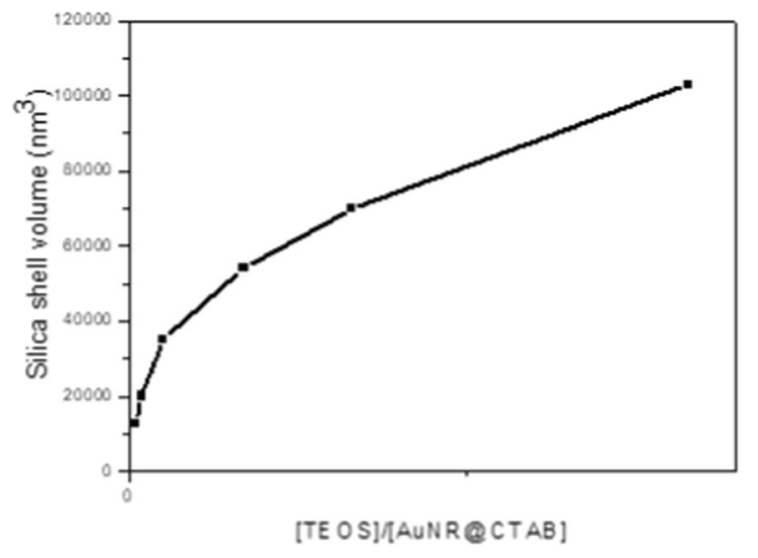

Fig. 12 Correlation between the silica shell volume of AuNR@ $\mathrm{SiO}_{2}$ with $[\mathrm{TEOS}] /[\mathrm{AuNR} @ \mathrm{CTAB}]$ ratio

to be more decisive for determining the thickness of the silica (Table 2). What appears to be decisive in controlling silica thickness is the [TEOS]/[AuNR@ CTAB] ratio (Fig. 12), while to monitor the actual growth of silica on AuNRs, the most suitable observable is the volume of silica shell, calculated from TEM images.

The thickness of the silica determines the position of the plasmon band of single and assembled gold nanorods. The effectiveness of the coupling depends on the mutual distance of the gold cores, which in turn depends on the thickness of the silica. In this perspective, using the silica shell as a spacer, it is possible to measure the edge-to-edge distance between the gold cores. By measuring the edge-to-edge distance between the gold cores of the examined samples, we found that it shortens by decreasing the silica thickness, thus allowing for better coupling. In particular, the coupling of the plasmonic fields is effective when the distance between the nanoparticles is not greater than $10 \mathrm{~nm}$, that is, in samples $3^{\prime}$ and $3^{\prime \prime}$, while if the distance is greater, no nanorods interact with each other and no difference is visible between solution and film spectra. The use of silica as a covering agent is an interesting way to regulate the plasmonic properties of gold nanorods. The longitudinal band moves towards the near-infrared as a function of the thickness of the shell, which determines the interaction between the gold cores and consequently the occurrence of plasmonic coupling. Consequently, goldcore/silica-shell nanorods are of considerable interest for a wide range of possible fields of application.
Acknowledgements The author, A.C., is grateful to Prof. Luis Liz-Marzan (Scientific director of CIC-Biomagune, Spain) for welcoming her into his laboratory and for her mentoring of nanoparticle synthesis techniques.

Funding Italian Ministry of University and Research supported this research through DEMETRA project (PON ARS01_00401).

\section{Declarations}

Conflict of interest The authors declare that they have no conflict of interest.

Open Access This article is licensed under a Creative Commons Attribution 4.0 International License, which permits use, sharing, adaptation, distribution and reproduction in any medium or format, as long as you give appropriate credit to the original author(s) and the source, provide a link to the Creative Commons licence, and indicate if changes were made. The images or other third party material in this article are included in the article's Creative Commons licence, unless indicated otherwise in a credit line to the material. If material is not included in the article's Creative Commons licence and your intended use is not permitted by statutory regulation or exceeds the permitted use, you will need to obtain permission directly from the copyright holder. To view a copy of this licence, visit http://creativecommons.org/licenses/by/4.0/.

\section{References}

Abadeer NS, Brennan MR, Wilson WL, Murphy CJ (2014) Distance and plasmon wavelength dependent fluorescence of molecules bound to silica-coated gold nanorods. 8392-8406

Adelt M, Maclaren DA, Birch DJS, Chen Y (2021) Morphological changes of silica shells deposited on gold nanorods: implications for nanoscale photocatalysts. ACS Applied Nano Materials 4:7730-7738. https://doi.org/10.1021/ acsanm.1c00977

Ahijado-Guzmán R, González-Rubio G, Izquierdo JG et al (2016) Intracellular pH-induced tip-to-tip assembly of gold nanorods for enhanced plasmonic photothermal therapy. ACS Omega 1:388-395. https://doi.org/10.1021/ acsomega.6b00184

Burda C, Chen X, Narayanan R, El-Sayed MA (2005) Chemistry and properties of nanocrystals of different shapes

Candreva A, Di Maio G, La Deda M (2020) A quick one-step synthesis of luminescent gold nanospheres. Soft Matter 16:10865-10868. https://doi.org/10.1039/d0sm02024a

Carrasco S, Benito-Peña E, Navarro-Villoslada F et al (2016) Multibranched gold-mesoporous silica nanoparticles coated with a molecularly imprinted polymer for labelfree antibiotic surface-enhanced raman scattering analysis. Chem Mater 28:7947-7954. https://doi.org/10.1021/acs. chemmater.6b03613 
Chen H, Shao L, Li Q, Wang J (2013) Gold nanorods and their plasmonic properties. Chem Soc Rev 42:2679-2724. https://doi.org/10.1039/c2cs35367a

Comenge J, Fragueiro O, Sharkey J et al (2016) Preventing plasmon coupling between gold nanorods improves the sensitivity of photoacoustic detection of labeled stem cells in vivo. ACS Nano 10:7106-7116. https://doi.org/10. 1021/acsnano.6b03246

Coursault D, Grand J, Zappone B et al (2012) Linear selfassembly of nanoparticles within liquid crystal defect arrays. Adv Mater 24:1461-1465. https://doi.org/10.1002/ adma.201103791

Creighton JA, Eadon DG (1991) Ultraviolet-visible absorption spectra of the colloidal metallic elements. J Chem Soc, Faraday Trans 87:3881-3891. https://doi.org/10.1039/ FT9918703881

Cretu C, Aa A, Candreva A et al (2018) Bisubstituted-biquinoline cu(i) complexes: synthesis, mesomorphism and photophysical studies in solution and condensed states. Journal of Materials Chemistry c 6:10073-10082. https://doi.org/10.1039/c8tc02999g

Eustis S, El-Sayed MA (2006) Why gold nanoparticles are more precious than pretty gold: noble metal surface plasmon resonance and its enhancement of the radiative and nonradiative properties of nanocrystals of different shapes. Chem Soc Rev 35:209-217. https://doi.org/10. 1039/b514191e

Gorelikov I, Matsuura N (2008) Single-step coating of mesoporous silica on cetyltrimethyl ammonium bromide-capped nanoparticles. Nano Lett 8:369-373. https://doi.org/10.1021/n10727415

Guerrero-Martínez A, Pérez-Juste J, Liz-Marzán LM (2010) Recent progress on silica coating of nanoparticles and related nanomaterials. Adv Mater 22:1182-1195. https://doi.org/10.1002/adma.200901263

Hao E, Schatz GC, Hupp JT (2004) Synthesis and optical properties of anisotropic metal nanoparticles. J Fluoresc 14:331-341. https://doi.org/10.1023/B:JOFL.00000 31815.71450.74

Ionescu A, Caligiuri R, Godbert N et al (2019) Electropolymerizable iriii complexes with $\beta$-ketoiminate ancillary ligands. Chem Asian J 14:3025-3034. https://doi.org/10. 1002/asia.201900521

Jain PK, Eustis S, El-Sayed MA (2006) Plasmon coupling in nanorod assemblies: optical absorption, discrete dipole approximation simulation, and exciton-coupling model. J Phys Chem B 110:18243-18253. https://doi.org/10. 1021/jp063879z

Jiang L, Mundoor H, Liu Q, Smalyukh II (2016) Electric switching of fluorescence decay in gold-silica-dye nematic nanocolloids mediated by surface plasmons. ACS Nano 10:7064-7072. https://doi.org/10.1021/acsna no.6b03216

Kawamura G, Yang Y, Nogami M (2007) Facile assembling of gold nanorods with large aspect ratio and their surface-enhanced Raman scattering properties. Appl Phys Lett 90:1-3. https://doi.org/10.1063/1.2752026

Kumar J, Wei X, Barrow S et al (2013) Surface plasmon coupling in end-to-end linked gold nanorod dimers and trimers. Phys Chem Chem Phys 15:4258-4264. https://doi. org/10.1039/c3cp44657c
Liz-Marzán LM, Giersig M, Mulvaney P (1996) Synthesis of nanosized gold-silica core-shell particles. Langmuir 12:4329-4335. https://doi.org/10.1021/la9601871

Lunn DJ, Finnegan JR, Manners I (2015) Self-assembly of "patchy" nanoparticles: a versatile approach to functional hierarchical materials. Chem Sci 6:3663-3673. https://doi. org/10.1039/c5sc01141h

Mayer KM, Hafner JH (2011) Localized surface plasmon resonance sensors. Chem Rev 111:3828-3857. https://doi.org/ $10.1021 / \mathrm{cr} 100313 \mathrm{v}$

Ming T, Zhao L, Yang Z, et al (2009) Strong polarization dependence of.pdf

Murphy CJ, Thompson LB, Chernak DJ et al (2011) Gold nanorod crystal growth: from seed-mediated synthesis to nanoscale sculpting. Curr Opin Colloid Interface Sci 16:128-134. https://doi.org/10.1016/j.cocis.2011.01.001

Ni W, Mosquera RA, Párez-Juste J, Liz-Marzán LM (2010) Evidence for hydrogen-bonding-directed assembly of gold nanorods in aqueous solution. J Phys Chem Lett 1:11811185. https://doi.org/10.1021/jz1002154

Pellas V, Hu D, Mazouzi Y, et al (2020) Gold nanorods for 1spr biosensing: synthesis, coating by silica, and bioanalytical applications

Pérez-Juste J, Pastoriza-Santos I, Liz-Marzán LM, Mulvaney P (2005) Gold nanorods: synthesis, characterization and applications. Coord Chem Rev 249:1870-1901. https:// doi.org/10.1016/j.ccr.2005.01.030

Pf L, Ghedini M, La Deda M et al (2020) Electrochromic behaviour of ir(iii) bis-cyclometalated 1,2-dioxolene tetrahalo complexes: fully reversible catecholate/semiquinone redox switches. Dalton Trans 49:2628-2635. https://doi. org/10.1039/c9dt04848k

Rožič B, Fresnais J, Molinaro C et al (2017) Oriented gold nanorods and gold nanorod chains within smectic liquid crystal topological defects. ACS Nano 11:6728-6738. https://doi.org/10.1021/acsnano.7b01132

Sahu AK, Raj S Effect of plasmonic coupling in different assembly of gold nanorods

Scarabelli L, Grzelczak M, Liz-Marzán LM (2013) Tuning gold nanorod synthesis through prereduction with salicylic acid. Chem Mater 25:4232-4238. https://doi.org/10. 1021/cm402177b

Scarabelli L, Sánchez-Iglesias A, Pérez-Juste J, Liz-Marzán LM (2015) A "Tips and Tricks" practical guide to the synthesis of gold nanorods. J Phys Chem Lett 6:4270-4279. https://doi.org/10.1021/acs.jpclett.5b02123

Teranishi T, Kurita R, Miyake M (2000) Shape control of Pt nanoparticles. J Inorg Organomet Polym Mater 10:145156. https://doi.org/10.1023/A:1009476128466

Vial S, Pastoriza-Santos I, Pérez-Juste J, Liz-Marzán LM (2007) Plasmon coupling in layer-by-layer assembled gold nanorod films. Langmuir 23:4606-4611. https://doi.org/ 10.1021/la063753t

Wang Y, Long S, Vdović S, Wang X (2013a) Fine tuning of the longitudinal plasmon resonance of gold nanorods by depleting gold precursor. Chem Mater 25:1372-1376. https://doi.org/10.1021/cm301832d

Wang F, Cheng S, Bao Z, Wang J (2013b) Anisotropic overgrowth of metal heterostructures induced by a site-selective silica coating. Angewandte Chemie - International 
Edition 52:10344-10348. https://doi.org/10.1002/anie. 201304364

Wang S, Lin Q, Xu W et al (2020) Precisely tuning the longitudinal localized surface plasmon resonance of gold nanorods: Via additive-regulated overgrowth. RSC Adv 10:12619-12625. https://doi.org/10.1039/d0ra00579g

Wu WC, Tracy JB (2015) Large-scale silica overcoating of gold nanorods with tunable shell thicknesses. Chem Mater 27:2888-2894. https://doi.org/10.1021/cm504764v

Wu J, Xiang D, Gordon R (2016) Characterizing gold nanorods in aqueous solution by acoustic vibrations probed with four-wave mixing. Opt Express 24:12458. https://doi.org/ 10.1364/oe.24.012458

Xia BY, Yang P, Sun Y, et al (2003) One-dimensional nanostructures: synthesis, characterization, and applications**. 353-389
Yamada S, Niidome Y (2006) Chapter 14 Gold nanorods: preparation, characterization, and applications to sensing and photonics. Handai Nanophotonics 2:255-274. https://doi. org/10.1016/S1574-0641(06)80020-3

Zhang Y, Liu Q, Mundoor H et al (2015) Metal nanoparticle dispersion, alignment, and assembly in nematic liquid crystals for applications in switchable plasmonic color filters and E-polarizers. ACS Nano 9:3097-3108. https:// doi.org/10.1021/nn5074644

Publisher's Note Springer Nature remains neutral with regard to jurisdictional claims in published maps and institutional affiliations. 